\title{
Long Term Transients in MOSFET 1/f Noise with Switched Bias
}

\author{
M.Y. Louie ${ }^{\#}$ and L. Forbes* \\ School of Electrical Engineering and Computer Science, Oregon State University, OR, USA
}

\begin{abstract}
Long term time dependent transients in 1/f noise have been observed and are reported on NMOS transistors operating with switched gate bias. The results are interpreted as a modification of the time dependence of random telegraph signals. The results have important implications in the understanding of the nature of $1 / \mathrm{f}$ noise and in the understanding the effect of $1 / f$ noise in switched capacitor and RF circuits with large amplitude switching gate voltages.
\end{abstract}

Keywords: Low frequency noise, noise transient analysis, phase noise, radio frequency (RF) circuits, switched bias circuits, switched capacitor circuits.

\section{INTRODUCTION}

Klumperink et al. [1], have recently had a number of publications on the low frequency noise of n-channel MOSFET's under switched gate bias conditions. Previously we investigated the same type of devices, older 5 micron commercial devices, and independently confirmed these prior results under the same type of switched bias conditions. There is an anomalous reduction in $1 / \mathrm{f}$ noise at frequencies two orders of magnitude below the switching frequency [2]. This reduction occurs only when holes are accumulated at the surface under the gate when the NMOS transistor is off during the switched bias. In previous work we used the same type of circuit as Klumperink et al., a differential circuit where two transistors are driven with the same gate signal and a differential output taken at the drains of the transistors. This leads to confusion over the result of the noise being $6 \mathrm{dBV}$ lower with switched bias. This reduction is due to the switched bias and both transistors being on only one half of the time. Instead we use a test circuit with a transistor substitution technique, depicted in Fig. (1), where two transistors are driven with complementary gate signals. One transistor is always on, even with switched bias. So the $1 / f$ noise should always stay the same level, independent of the switched bias. However as shown in Fig. (2) there is again an anomalous reduction in the $1 / \mathrm{f}$ noise of $6 \mathrm{dBV}$ or more at frequencies one and two orders of magnitude below the switching frequency. This work follows earlier reports of switched bias effects on $1 /$ f noise $[3,4]$.

\section{TRANSIENTS IN 1/F NOISE}

More recently we investigated the time dependence of switched bias $1 / \mathrm{f}$ noise and have discovered long term time dependent transients in the $1 / \mathrm{f}$ noise $[5,6]$. We describe here a detailed investigation of the long term transients in $1 / \mathrm{f}$ noise under switched bias conditions. We have now used a test circuit with a transistor substitution technique, depicted in Fig. (1), where two transistors are driven with complementary gate signals. One transistor is always on, even with

*Address correspondence to this author at the School of Electrical Engineering and Computer Science, Oregon State University, Corvallis, OR 973315501, USA; Tel: 541-753-1409; E-mail: prof @forbes4.com

"M. Y. Louie is now with the Advanced Pixel Research Lab, MagnaChip, Lake Oswego, OR, USA.

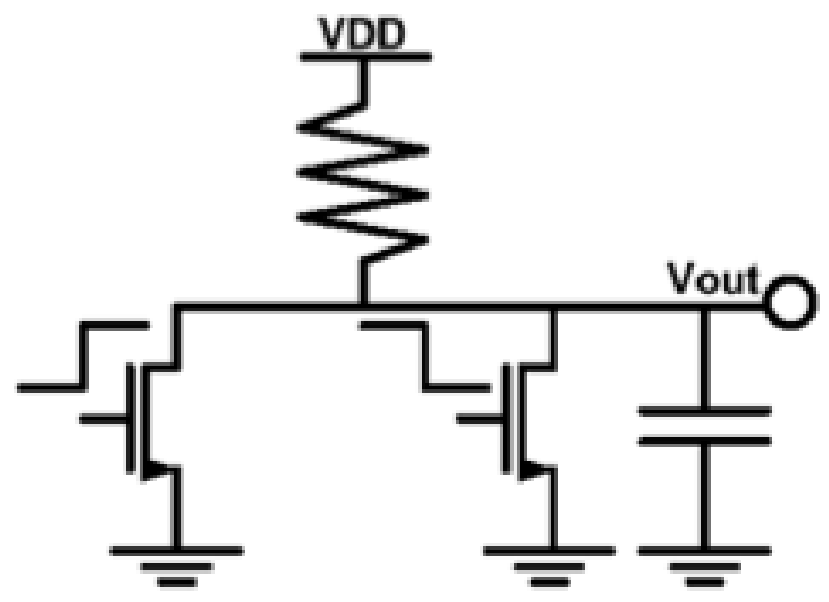

Fig. (1). Test circuit using a transistor substitution technique used in fixed and switched bias.

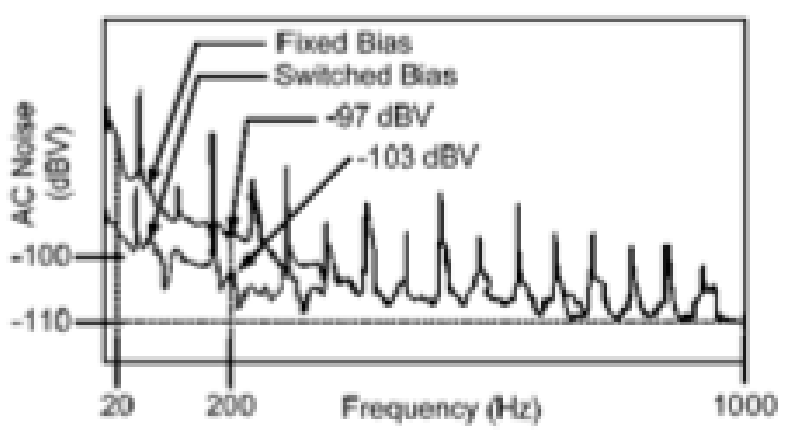

Fig. (2). Reduction in $1 / \mathrm{f}$ noise at low frequencies with switched bias, $2 \mathrm{kHz}$ switching frequency using test circuit.

switched bias so the $1 / \mathrm{f}$ noise should always be the same independent of the time of the measurement, and independent of the switched bias. A filter circuit is used to measure the noise at a fixed frequency of $1 \mathrm{kHz}$ with a $100 \mathrm{kHz}$ switched bias. When switched bias is first applied the noise stepped down as previously been observed, depicted in Fig. (3). This initial step down in $1 / f$ noise during application of switched bias is observable only if the initial gate voltage on the transistors is near zero bias. Otherwise, this step is not observed in cases where the bias voltage in the off condition is near threshold $[1,2]$ show in Fig. (4). This reduction in 1/f noise 
depends on whether holes accumulate at the surface during the off condition. If the gate bias during the off condition with switched bias is near threshold, depicted in Fig. (4), there is only a small or no reduction in the $1 / f$ noise with switched bias. The $1 / f$ noise always returns to the same level or value with fixed bias. Similar phenomena have been observed on JFET devices although at a very much lower frequency, indicative of their much lower surface area.

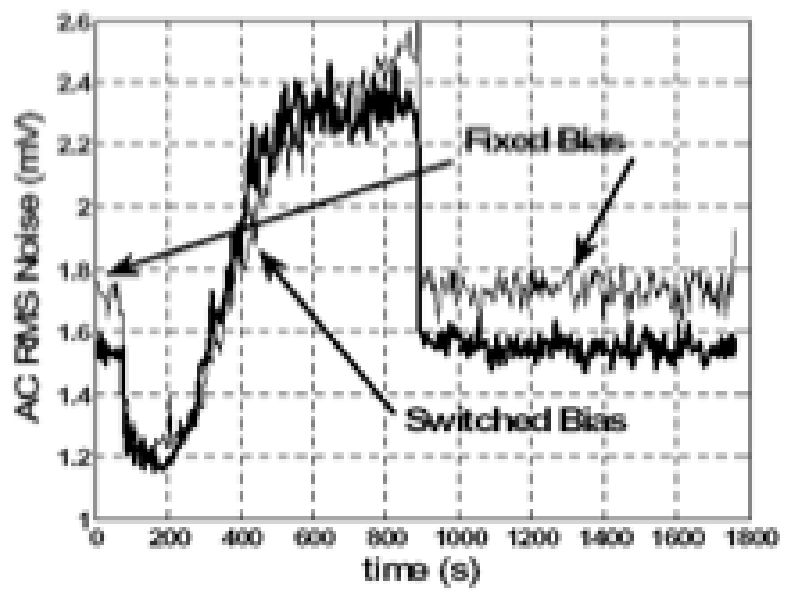

Fig. (3). Noise at $1 \mathrm{kHz}$ with fixed bias initially, then switched bias for 15 minutes, then fixed bias for 15 minutes, off gate bias voltage is zero; two different devices.

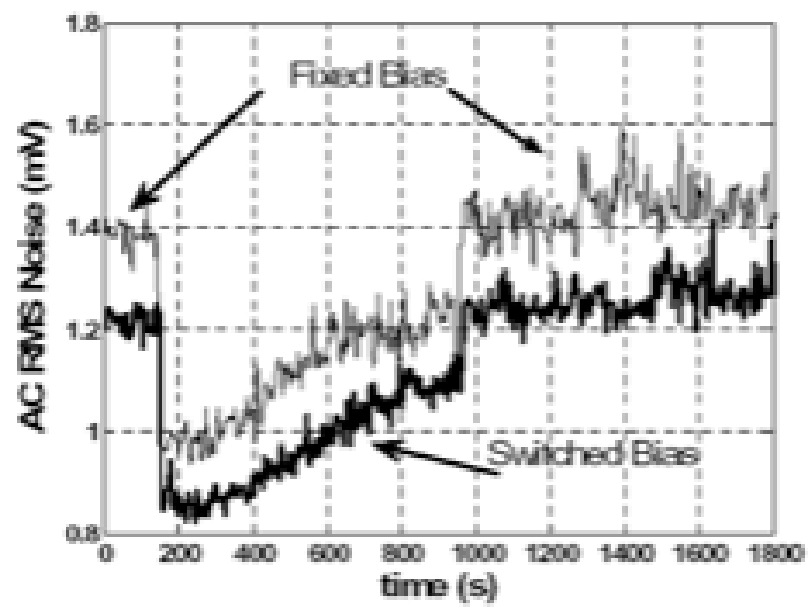

Fig. (4). Noise at $1 \mathrm{kHz}$ with fixed bias, then switched bias for 15 minutes, then fixed bias for 15 minutes. Off gate bias voltage is near threshold; for the same two devices that appeared in Fig. (3).

A variety of different types of transient behavior shown in Fig. (5), have been observed on devices from two different manufacturers obtained over different time periods years apart. In some cases the $1 / \mathrm{f}$ noise will decrease after the application of fixed bias and stay at a lower level for long periods of time, however, the next time switched bias is applied it will recover from this lower level. In other cases the $1 / f$ noise will initially decrease with switched bias but recover in shorter periods of time. Long term transient effects are observed in the transients as depicted in Fig. (5). In these cases the observed $1 / \mathrm{f}$ noise recovered close to or above the fixed bias level after initial reduction from switched bias, then dips down and eventually decays back down below the fixed bias noise level. The time where $1 / \mathrm{f}$ noise recovered and stayed above the fixed bias level before dipping down is also influenced by the detailed occupancy of the traps.

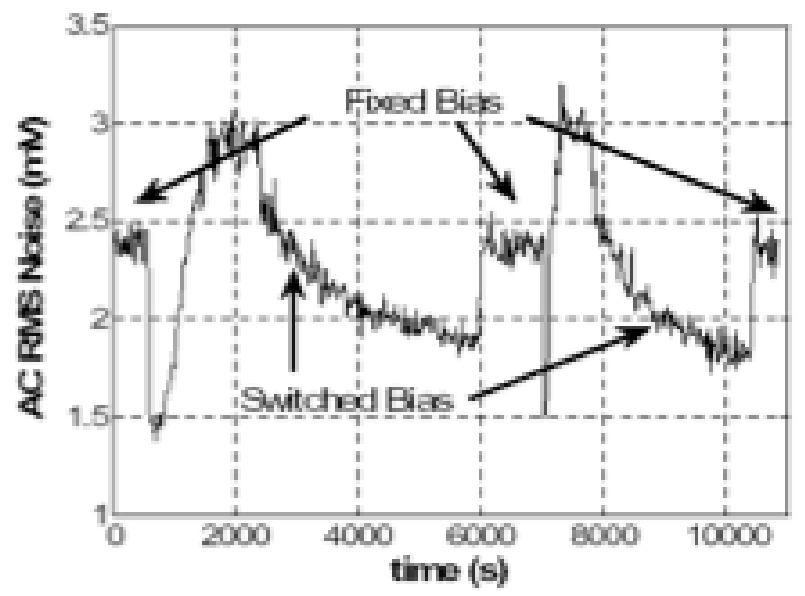

Fig. (5). Noise at $1 \mathrm{kHz}$ with fixed bias, then switched bias for 100 minutes, fixed bias for 15 minutes, then switched bias for 50 minutes, and finally fixed bias; note the wear out or annealing in the second switched bias sequences and faster recovery from the lower initial noise level. There are a variety of different transients observed over long time periods on devices from two manufacturers.

\section{PHYSICAL PHENOMENA}

One theory for the origin of 1/f noise in MOSFET's is the superposition of multiple independent random telegraph signals or the RTS model [7]. The capture and release of electrons in the oxide traps is described below (1), and illustrated in Fig. (6).

$$
\mathrm{S}(f) \approx\left(k T A t_{o x} N_{t}\right) /\left(2 f \log \left(\lambda_{\mathrm{H}} / \lambda_{\mathrm{L}}\right)\right) .
$$

where, $\mathrm{k}$, is Boltzman's constant, $\mathrm{T}$, is the temperature in degrees Kelvin, $\mathrm{A}$, is the device area, $t_{\mathrm{ox}}$, is the gate oxide thickness, $\mathrm{f}$, frequency and $\mathrm{N}_{\mathrm{t}}$ the trap density per unit volume. This yields the power spectral density in power, watts, per $\mathrm{Hz}^{2}$. 1/f noise depends on the slowest transition rate $\lambda_{\mathrm{L}}$. $1 / \lambda_{\mathrm{L}}$ is the time between slow oxide trapping events. At the beginning of switched bias the slow traps are not kept filled, $\lambda_{\mathrm{L}}$ becomes smaller, and the log of the ratio of highest and lowest transition rate becomes larger. Overall 1/f noise decreases as is evident from time domain plots in Fig. (3). However, repeated electron inversion at the surface with switched bias causes the transition rates to change. As slowest ones become faster, $\lambda_{\mathrm{L}}$ increases and the $\log$ of the ratio of the highest and lowest transition rates decreases. This results in 1/f noise increasing over time, as depicted in Fig. (5).

1/f noise and its anomalous reduction is still observed even at a lower temperature, $77 \mathrm{~K}$, but with a slightly smaller magnitude. From Eqn. 1 temperature affects the 1/f noise directly as, $\mathrm{T}$, but at low temperature the lowest transition rate becomes very small or the time constant becomes very long. Temperature has been observed to affect the $1 / \mathrm{f}$ noise to a lesser degree as compared to switched bias.

Scaling exacerbates the effects of $1 / f$ noise on the circuit since $1 / f$ noise power is independent of the device dimensions but the signal power decreases with device and technology scaling. As modern MOSFET transistors are scaled down in size with the same width to length ratio, W/L, the 
drain current remains the same or can actually decrease and lower voltages are used resulting in lower signal power. The signal to noise power decreases with scaling.
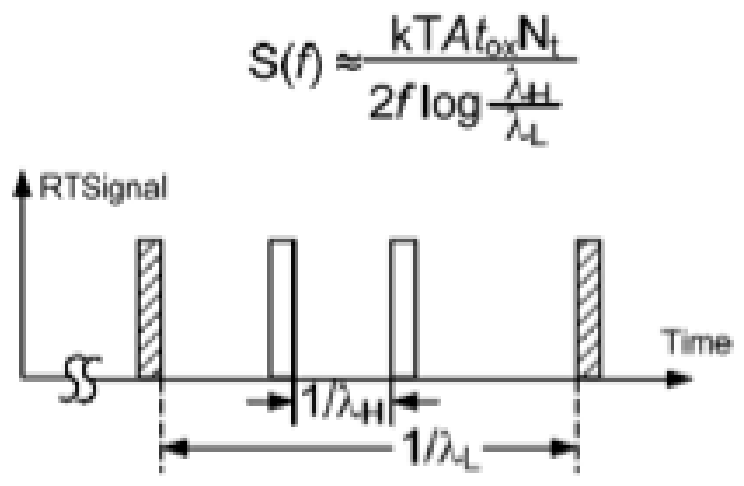

Fig. (6). Random telegraph signal model for $1 / \mathrm{f}$ noise. $S(f)$ is modeled by the superposition of multiple random telegraph signals, RTS.

\section{CONCLUSIONS}

These transient phenomena in 1/f noise, overlooked for 40 years, could provide important clues regarding the nature and causes of $1 / f$ noise. Modern electronic circuits seldom employ transistors under D.C. fixed bias conditions with small A.C. signals superimposed. More often than not the small signal 1/f and white noise of the transistor are superimposed upon the large signal switched capacitor or RF signals. Circuit simulators currently use fixed DC bias noise models which are not representative of the actual operating conditions, particularly since switched bias phenomena have been observed in $1 / \mathrm{f}$ noise.

\section{REFERENCES}

[1] P. van der Wel, E. A. M. Klumperink, S. L. J Gierkink, R. F. Wassenaar, and H. Wallinga, "MOSFET 1/f Noise Measurement Under Switched Bias Conditions", IEEE Electron Device Lett., vol. 21, no. 1, pp. 43-46, Jan 2000

[2] M. B. Weissmann, " $1 / \mathrm{f}$ Noise and other slow, nonexponential kinetics in condensed matter", Rev. Mod. Phys., vol. 60, no. 2, pp. 537-571, Apr 1988.

[3] I. Bloom and Y. Nemirovsky, "1/f Noise reduction of metal-oxide semiconductor transistors by cycling from inversion to accumulation”, Appl. Phys. Lett., vol. 58, pp. 1664-1666, Apr 1991.

[4] M.Y. Louie and L. Forbes, "MOSFET 1/f noise under switched bias conditions", Proc. 3rd Int. Symp. On Fluct. and Noise, Austin, TX, 2005, pp. 215-225.

[5] M.Y. Louie, L. Forbes, "Long term transients in MOSFET 1/f noise under switched bias conditions ", Workshop on Microelectronics and Electron Devices, Boise, Idaho, 2005, pp. 55-58.

[6] M.Y. Louie, D.A. Miller, M.E. Jacobs and L. Forbes, "Long term transients in MOSFET 1/f noise with switched bias", Abst. IEEE Device Research Conf., Santa Barbara CA, 2005, pp. 79-80.

[7] H. Tian and A. E. Gamal, "Analysis of 1/f noise in CMOS APS," Proceedings of the SPIE - The International Society for Optical Engineering, vol. 3965, 2000. pp.168-76. 